\title{
A comparison of younger $v$. older adults' ability to estimate food portion sizes
}

\author{
C. M. Timon ${ }^{1}$, S. E. Forster ${ }^{1}$, M. E. Barker ${ }^{1}$, A. J. Godfrey ${ }^{3}$, F. Hwang ${ }^{2}$ and E. A. Williams ${ }^{1}$ \\ ${ }^{1}$ Human Nutrition Unit, Department of Oncology, University of Sheffield, Sheffield S10 2RX, ${ }^{2}$ School of Systems \\ Engineering, University of Reading, Reading, RG6 6AY and ${ }^{3}$ Clinical Ageing Research Unit, Newcastle University, \\ Newcastle upon Tyne, NE4 SPL
}

In dietary studies, it is important to have accurate information on food intake at both an individual and population level. For many methods of dietary assessment, a food portion size must be identified for each food item consumed in order to calculate the corresponding nutrient value of each food item. The Food Atlas of Portion Sizes ${ }^{(1)}$ is a commonly used tool for the identification of portion sizes of foods consumed. Participants are asked to select a portion size photograph from the Food Atlas that most accurately represents the portion size of a food they have consumed.

Forty older adults (65 years + ) and forty-one younger adults (18-40 years) were recruited and asked to assess the portion size of eighteen self-served food portions. The foods were chosen on the basis that they were commonly consumed by older adults ${ }^{(2)}$ and also represented a range of food morphologies. Participants were shown eight food portion size photographs for each food by means of the photographs from the Food Atlas or by viewing the same food photographs on a computer screen. Participants were asked to select the photograph that most accurately represented the food portion they had served. The self-served portions were weighed before the portion size assessment so a comparison of actual $v$. estimated weight could be made. The eighteen different foods were grouped into five food morphology categories (amorphous, small pieces, large pieces, shaped and spread) and the accuracy of portion size assessment was compared by age group and whether photographs had been shown traditionally or on a computer screen (method). This study was approved by the University of Sheffield, School of Medicine Research Ethics Committee.

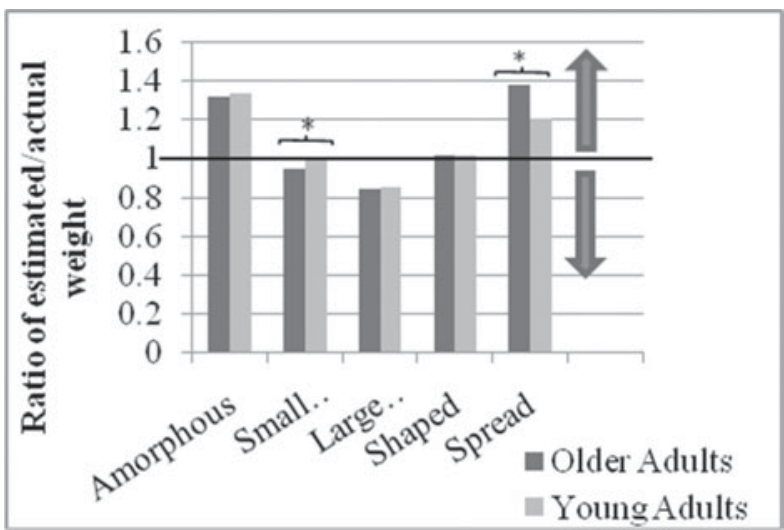

Fig. 1. The effect of age on portion size assessment.

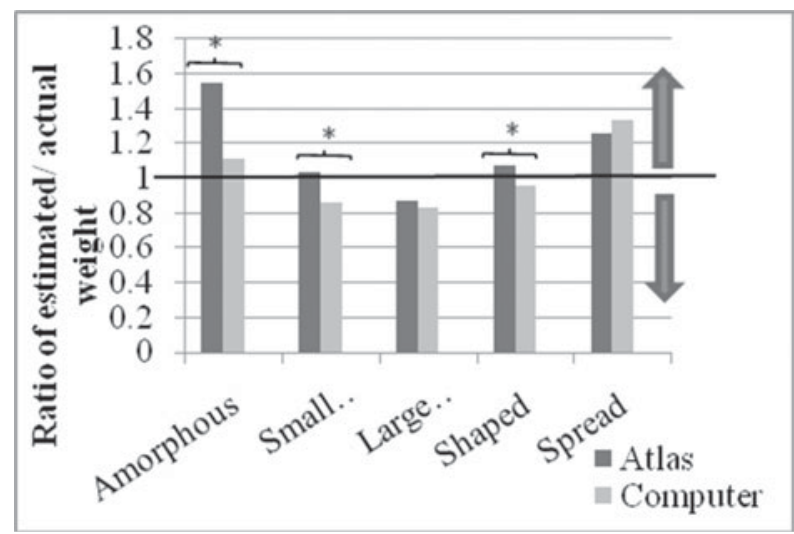

Fig. 2. The effect of method on portion size assessment.

Statistical analysis was carried out using ANOVA to examine the effect of age (Fig. 1) and method (Fig. 2) on the ability to estimate the portion size. A value over 1 denotes overestimation and a value below 1 denotes underestimation. There was no interaction between age and method in portion size assessment however, there were significant $(P<0.05)$ differences in the ability of older and younger adults to assess portion size. Older adults significantly overestimated spreads and underestimated small pieces. The traditional food atlas method was significantly different from the computer method for estimation of amorphous, small pieces and shaped food morphologies. The reason for this is unknown, although the computer-based method displayed only two photographs at a time that may alter the portion size estimation of certain foods. This study suggests that older adults may be less able than younger adults to estimate portion size of certain food categories, and highlights the need for improved dietary assessment methodologies.

1. Nelson M, Atkinson M \& Meyer J (1997) A Photographic Atlas of Food Portion Sizes. London: MAFF Publications.

2. Finch S, Doyle W, Lowe C et al. (1998) National Diet and Nutrition Survey: People Aged 65 Years and Over. Report of the Diet and Nutrition Survey. London: The Stationary Office. 